TAIWANESE JOURNAL OF MATHEMATICS

Vol. 15, No. 3, pp. 961-980, June 2011

This paper is available online at http://www.tjm.nsysu.edu.tw/

\title{
WEAK AND STRONG CONVERGENCE THEOREMS FOR POSITIVELY HOMOGENEOUS NONEXPANSIVE MAPPINGS IN BANACH SPACES
}

\author{
Wataru Takahashi and Jen-Chih Yao*
}

\begin{abstract}
Our purpose in this paper is first to prove a weak convergence theorem by Mann's iteration for positively homogeneous nonexpansive mappings in a Banach space. Further, using the shrinking projection method defined by Takahashi, Takeuchi and Kubota, we prove a strong convergence theorem for such mappings. From two results, we obtain weak and strong convergence theorems for linear contractive mappings in a Banach space. These results are new even if the mappings are linear and contractive.
\end{abstract}

\section{INTRODUCTION}

Let $H$ be a real Hilbert space with inner product $\langle\cdot, \cdot\rangle$ and norm $\|\cdot\|$ and let $C$ be a closed convex subset of $H$. Let $T$ be a mapping of $C$ into itself. Then we denote by $F(T)$ the set of fixed points of $T$. A mapping $T: C \rightarrow C$ is called nonexpansive if $\|T x-T y\| \leq\|x-y\|$ for all $x, y \in C$. From [46] we know a weak convergence theorem by Mann's iteration for nonexpansive mappings in a Hilbert space:

Let $T: C \rightarrow C$ be a nonexpansive mapping with $F(T) \neq \emptyset$ and define a sequence $\left\{x_{n}\right\}$ in $C$ by $x_{1}=x \in C$ and

$$
x_{n+1}=\alpha_{n} x_{n}+\left(1-\alpha_{n}\right) T x_{n}, \quad \forall n \in \mathbb{N},
$$

where $\left\{\alpha_{n}\right\}$ is a real sequence in $[0,1]$ such that

$$
\sum_{n=1}^{\infty} \alpha_{n}\left(1-\alpha_{n}\right)=\infty .
$$

Then, $\left\{x_{n}\right\}$ converges weakly to an element $z$ of $F(T)$, where $z=\lim _{n \rightarrow \infty} P x_{n}$ and $P$ is the metric projection of $H$ onto $F(T)$. By Reich [36], such a theorem

Received November 9, 2009.

2000 Mathematics Subject Classification: 47H05, 47H09, 47H20.

Key words and phrases: Banach space, Nonexpansive mapping, Fixed point, Generalized nonexpansive mapping, Hybrid method, Mann's iteration.

*Corresponding author. 
was extended to a uniformly convex Banach space with a Fréchet differentiable norm. However, we do not know whether the fixed point $z$ is characteraized under any projections in a Banach space. On the other hand, Nakajo and Takahashi [33] proved a strong convergence theorem for nonexpansive mappings in a Hilbert space by using the hybrid method in mathematical programming:

Let $T: C \rightarrow C$ be a nonexpansive mapping with $F(T) \neq \emptyset$ and let $\left\{\alpha_{n}\right\}$ be a real sequence in $[0,1]$ such that $0 \leq \alpha_{n} \leq a<1$ for all $n \in \mathbb{N}$. Define a sequence $\left\{x_{n}\right\}$ in $C$ by $x_{1}=x \in C$ and

$$
\left\{\begin{array}{l}
u_{n}=\alpha_{n} x_{n}+\left(1-\alpha_{n}\right) T x_{n}, \\
C_{n}=\left\{z \in C:\left\|u_{n}-z\right\| \leq\left\|x_{n}-z\right\|\right\} \\
Q_{n}=\left\{z \in C:\left\langle x_{n}-z, x-x_{n}\right\rangle \geq 0\right\} \\
x_{n+1}=P_{C_{n} \cap Q_{n}} x, \quad \forall n \in \mathbb{N},
\end{array}\right.
$$

where $P_{C_{n} \cap Q_{n}}$ is the metric projection of $H$ onto $C_{n} \cap Q_{n}$. Then, $\left\{x_{n}\right\}$ converges strongly to an element $z$ of $F(T)$, where $z=P_{F(T)} x$ and $P_{F(T)}$ is the metric projection of $H$ onto $F(T)$. However, we do not know whether such a strong convergence theorem for nonexpansive mappings is extended to a Banach space. Many authors have extended this convergence theorem to a Banach space by using nonlinear mappings which are different from a nonexpansive mapping; see, for instance, [28].

Our purpose in this paper is first to prove a weak convergence theorem by Mann's iteration for positively homogeneous nonexpansive mappings in a Banach space. In the theorem, the limit of weak convergence is characteraized by using a sunny generalized nonexpansive retraction in Ibaraki and Takahashi [14]. Further, using the shrinking projection method defined by Takahashi, Takeuchi and Kubota, we prove a strong convergence theorem for positively homogeneous nonexpansive mappings in a Banach space. From two results, we obtain weak and strong convergence theorems for linear contractive mappings in a Banach space. These results are new even if the mappings are linear and contractive.

\section{PReliminaries}

Let $E$ be a real Banach space with norm $\|\cdot\|$ and let $E^{*}$ be the dual of $E$. We denote the value of $y^{*} \in E^{*}$ at $x \in E$ by $\left\langle x, y^{*}\right\rangle$. When $\left\{x_{n}\right\}$ is a sequence in $E$, we denote the strong convergence of $\left\{x_{n}\right\}$ to $x \in E$ by $x_{n} \rightarrow x$ and the weak convergence by $x_{n} \rightarrow x$. The modulus $\delta$ of convexity of $E$ is defined by

$$
\delta(\epsilon)=\inf \left\{1-\frac{\|x+y\|}{2}:\|x\| \leq 1,\|y\| \leq 1,\|x-y\| \geq \epsilon\right\}
$$


for every $\epsilon$ with $0 \leq \epsilon \leq 2$. A Banach space $E$ is said to be uniformly convex if $\delta(\epsilon)>0$ for every $\epsilon>0$. A uniformly convex Banach space is strictly convex and reflexive. Let $C$ be a nonempty subset of a Banach space $E$. A mapping $T: C \rightarrow C$ is nonexpansive if $\|T x-T y\| \leq\|x-y\|$ for all $x, y \in C$. A mapping $T: C \rightarrow C$ is quasi-nonexpansive if $F(T) \neq \emptyset$ and $\|T x-y\| \leq\|x-y\|$ for all $x \in C$ and $y \in F(T)$, where $F(T)$ is the set of fixed points of $T$. If $C$ is a closed convex subset of $E$ and $T: C \rightarrow C$ is quasi-nonexpansive, then $F(T)$ is closed and convex; see Itoh and Takahashi [17]. From [44] we know the following lemma.

Lemma 2.1. Let $E$ be a uniformly convex Banach space and let $\delta$ be the modulus of convexity in $E$. Let $0<\epsilon \leq 2 r$. Then, $\delta\left(\frac{\epsilon}{r}\right)>0$ and

$$
\|\alpha x+(1-\alpha) y\| \leq r\left\{1-2 \alpha(1-\alpha) \delta\left(\frac{\epsilon}{r}\right)\right\}
$$

for all $x, y \in E$ with $\|x\| \leq r,\|y\| \leq r$ and $\|x-y\| \geq \epsilon$ and $\alpha \in[0,1]$.

Further, we know the following result by Browder; see [44].

Lemma 2.2. Let $E$ be a uniformly convex Banach space and let $C$ be a bounded closed convex subset of $E$. Let $T: C \rightarrow C$ be a nonexpansive mapping. If $\left\{x_{n}\right\}$ is a sequence of $C$ such that $x_{n} \rightarrow u$ and $x_{n}-T x_{n} \rightarrow 0$, then $u$ is a fixed point of $T$.

Let $C$ be a nonempty closed convex subset of a strictly convex and reflexive Banach space $E$. Then we know that for any $x \in E$, there exists a unique element $z \in C$ such that $\|x-z\| \leq\|x-y\|$ for all $y \in C$. Putting $z=P_{C}(x)$, we call $P_{C}$ the metric projection of $E$ onto $C$. The duality mapping $J$ from $E$ into $2^{E^{*}}$ is defined by

$$
J x=\left\{x^{*} \in E^{*}:\left\langle x, x^{*}\right\rangle=\|x\|^{2}=\left\|x^{*}\right\|^{2}\right\}
$$

for every $x \in E$. Let $U=\{x \in E:\|x\|=1\}$. The norm of $E$ is said to be Gâteaux differentiable if for each $x, y \in U$, the limit

$$
\lim _{t \rightarrow 0} \frac{\|x+t y\|-\|x\|}{t}
$$

exists. In the case, $E$ is called smooth. We know that $E$ is smooth if and only if $J$ is a single-valued mapping of $E$ into $E^{*}$. We also know that $E$ is reflexive if and only if $J$ is surjective, and $E$ is strictly convex if and only if $J$ is one-to-one. Therefore, if $E$ is a smooth, strictly convex and reflexive Banach space, then $J$ is a single-valued bijection and in this case, the inverse mapping $J^{-1}$ coincides with the duality mapping $J_{*}$ on $E^{*}$. The norm of $E$ is said to be uniformly Gateaux differentiable if for each $y \in U$, the limit (2.1) is attained uniformly for $x \in U$. It 
is also said to be Fréchet differentiable if for each $x \in U$, the limit (2.1) is attained uniformly for $y \in U$. A Banach space $E$ is called uniformly smooth if the limit (2.1) is attained uniformly for $x, y \in U$. It is known that if the norm of $E$ is uniformly Gâteaux differentiable, then $J$ is uniformly norm to weak* continuous on each bounded subset of $E$, and if the norm of $E$ is Frechet differentiable, then $J$ is norm to norm continuous. If $E$ is uniformly smooth, $J$ is uniformly norm to norm continuous on each bounded subset of $E$. For more details, see [44]. We know the following results; see [44].

Theorem 2.3. Let $E$ be a smooth, strictly convex and reflexive Banach space. Let $C$ be a nonempty closed convex subset of $E$ and let $P_{C}$ be the metric projection of $E$ onto $C$. Let $x_{0} \in C$ and $x_{1} \in E$. Then, $x_{0}=P_{C}\left(x_{1}\right)$ if and only if

$$
\left\langle x_{0}-y, J\left(x_{1}-x_{0}\right)\right\rangle \geq 0
$$

for all $y \in C$, where $J$ is the duality mapping of $E$.

Theorem 2.4. Let $E$ be a smooth Banach space and let $J$ be the duality mapping on $E$. Then, $\langle x-y, J x-J y\rangle \geq 0$ for all $x, y \in E$. Further, if $E$ is strictly convex and $\langle x-y, J x-J y\rangle=0$, then $x=y$.

Let $E$ be a reflexive, strictly convex and smooth Banach space. The function $\phi: E \times E \rightarrow(-\infty, \infty)$ is defined by

$$
\phi(x, y)=\|x\|^{2}-2\langle x, J y\rangle+\|y\|^{2}
$$

for $x, y \in E$, where $J$ is the duality mapping of $E$; see [1] and [21]. We have from the definition of $\phi$ that

$$
\phi(x, y)=\phi(x, z)+\phi(z, y)+2\langle x-z, J z-J y\rangle
$$

for all $x, y, z \in E$. From $\left(\|x\|^{2}-\|y\|^{2}\right) \leq \phi(x, y)$ for all $x, y \in E$, we can see that $\phi(x, y) \geq 0$. Let $\phi_{*}: E^{*} \times E^{*} \rightarrow(-\infty, \infty)$ be the function defined by

$$
\phi_{*}\left(x^{*}, y^{*}\right)=\left\|x^{*}\right\|^{2}-2\left\langle J^{-1} y^{*}, x^{*}\right\rangle+\left\|y^{*}\right\|^{2}
$$

for $x^{*}, y^{*} \in E^{*}$, where $J$ is the duality mapping of $E$. It is easy to see that

$$
\phi(x, y)=\phi_{*}(J y, J x)
$$

for $x, y \in E$. If $E$ is additionally assumed to be strictly convex, then

$$
\phi(x, y)=0 \Longleftrightarrow x=y .
$$


If $C$ is a nonempty closed convex subset of a smooth, strictly and reflexive Banach space $E$, then for all $x \in E$ there exists a unique $z \in C$ (denoted by $\Pi_{C} x$ ) such that

$$
\phi(z, x)=\min _{y \in C} \phi(y, x) .
$$

The mapping $\Pi_{C}$ is called the generalized projection from $E$ onto $C$; see Alber [1], Alber and Reich [2], and Kamimura and Takahashi [21]. The following lemmas are well known; see, for instance, [21].

Lemma 2.5. Let $E$ be a uniformly convex and smooth Banach space and let $\left\{x_{n}\right\}$ and $\left\{y_{n}\right\}$ be sequences in $E$ such that $\left\{x_{n}\right\}$ or $\left\{y_{n}\right\}$ is bounded. If $\lim _{n \rightarrow \infty} \phi\left(x_{n}, y_{n}\right)=0$, then $\lim _{n \rightarrow \infty}\left\|x_{n}-y_{n}\right\|=0$.

Lemma 2.6. Let $E$ be a uniformly convex and smooth Banach space and let $r>0$. Then, there exists a strictly increasing, continuous and convex function $g:[0, \infty) \rightarrow[0, \infty)$ such that $g(0)=0$ and

$$
g(\|x-y\|) \leq \phi(x, y)
$$

for all $x, y \in B_{r}$, where $B_{r}=\{z \in E:\|z\| \leq 0\}$.

For a sequence $\left\{C_{n}\right\}$ of nonempty closed convex subsets of a reflexive Banach space $E$, define s- $-\mathrm{Li}_{n} C_{n}$ and w-Ls $C_{n}$ as follows: $x \in \mathrm{s}-\mathrm{Li}_{n} C_{n}$ if and only if there exists $\left\{x_{n}\right\} \subset E$ such that $\left\{x_{n}\right\}$ converges strongly to $x$ and that $x_{n} \in C_{n}$ for all $n \in \mathbb{N}$. Similarly, $y \in \mathrm{w}-\mathrm{Ls}_{n} C_{n}$ if and only if there exists a subsequence $\left\{C_{n_{i}}\right\}$ of $\left\{C_{n}\right\}$ and a sequence $\left\{y_{i}\right\} \subset E$ such that $\left\{y_{i}\right\}$ converges weakly to $y$ and that $y_{i} \in C_{n_{i}}$ for all $i \in \mathbb{N}$. If $C_{0}$ satisfies that

$$
C_{0}=\mathrm{s}-\mathrm{Li}_{n} C_{n}=\mathrm{w}-\mathrm{Ls}_{n} C_{n},
$$

it is said that $\left\{C_{n}\right\}$ converges to $C_{0}$ in the sense of Mosco [30] and we write $C_{0}=\mathrm{M}-\lim _{n \rightarrow \infty} C_{n}$. It is easy to show that if $\left\{C_{n}\right\}$ is nonincreasing with respect to inclusion, then $\left\{C_{n}\right\}$ converges to $\cap_{n=1}^{\infty} C_{n}$ in the sense of Mosco. For more details, see [30]. We know the following theorem [12].

Theorem 2.7. Let $E$ be a smooth Banach space and let $E^{*}$ have a Fréchet differentiable norm. Let $\left\{C_{n}\right\}$ be a sequence of nonempty closed convex subsets of $E$. If $C_{0}=\mathrm{M}-\lim _{n \rightarrow \infty} C_{n}$ exists and nonempty, then for each $x \in E, \Pi_{C_{n}} x$ converges strongly to $\Pi_{C_{0}} x$, where $\Pi_{C_{n}}$ and $\Pi_{C_{0}}$ are the generalized projections of $E$ onto $C_{n}$ and $C_{0}$, respectively. 
Let $E$ be a Banach space and let $D$ be a nonempty closed subset of $E$. A mapping $R: E \rightarrow D$ is said to be sunny if

$$
R(R x+t(x-R x))=R x, \quad \forall x \in E, \forall t \geq 0 .
$$

A mapping $R: E \rightarrow D$ is a retraction if $R x=x$ for all $x \in D$. A nonempty subset of a smooth Banach space $E$ is said to be a generalized nonexpansive retract (resp. sunny generalized nonexpansive retract) of $E$ if there exists a generalized nonexpansive retraction (resp. sunny generalized nonexpansive retraction) of $E$ onto $D$. From [14], we know the following lemmas.

Lemma 2.8. (Ibaraki and Takahashi [14]). Let $E$ be a smooth, strictly convex and reflexive Banach space and let $D$ be a nonempty closed subset of $E$. Then, a sunny generalized nonexpansive retraction of $E$ onto $D$ is uniquely determined.

Lemma 2.9. (Ibaraki and Takahashi [14]). Let $E$ be a smooth, strictly convex and reflexive Banach space and let $D$ be a nonempty closed subset of $E$. Suppose that there exists a sunny generalized nonexpansive retraction $R$ of $E$ onto $D$ and let $(x, z) \in E \times D$. Then, the following hold:

(1) $z=R x$ if and only if $\langle x-z, J y-J z\rangle \leq 0, \forall y \in D$;

(2) $\phi(R x, z)+\phi(x, R x) \leq \phi(x, z)$.

In 2007, Kohsaka and Takahashi [24] proved the following results.

Lemma 2.10. (Kohsaka and Takahashi [24]). Let $E$ be a smooth, strictly convex and reflexive Banach space and let $C_{*}$ be a nonempty closed convex subset of $E^{*}$. Suppose that $\Pi_{C_{*}}$ is the generalized projection of $E^{*}$ onto $C_{*}$. Then, $R$ defined by $R=J^{-1} \Pi_{C_{*}} J$ is a sunny generalized nonexpansive retraction of $E$ onto $J^{-1} C_{*}$.

Lemma 2.11. (Kohsaka and Takahashi [24]). Let $E$ be a smooth, strictly convex and reflexive Banach space and let $D$ be a nonempty subset of $E$. Then, the following conditions are equivalent

(1) $D$ is a sunny generalized nonexpansive retract of $E$;

(2) $D$ is a generalized nonexpansive retract of $E$;

(3) $J D$ is closed and convex.

In this case, $D$ is closed.

Lemma 2.12. (Kohsaka and Takahashi [24]). Let $E$ be a smooth, strictly convex and reflexive Banach space and let $D$ be a nonempty closed subset of $E$. Suppose that there exists a sunny generalized nonexpansive retraction $R$ of $E$ onto $D$ and let $(x, z) \in E \times D$. Then, the following conditions are equivalent 
(1) $z=R x$;

(2) $\phi(x, z)=\min _{y \in D} \phi(x, y)$.

Let $E$ be a smooth Banach space $E$ and let $C$ be a nonempty subset of $E$. A mapping $T: C \rightarrow C$ is generalized nonexpansive [14] if $F(T) \neq \emptyset$ and

$$
\phi(T x, y) \leq \phi(x, y)
$$

for all $x \in C$ and $y \in F(T)$. From Ibaraki and Takahashi [15] we know the following lemma.

Lemma 2.13. (Ibaraki and Takahashi [15]). Let $E$ be a smooth, strictly convex and reflexive Banach space and let $T$ be a generalized nonexpansive mapping of $E$ into itself. Then, $F(T)$ is a sunny generalized nonexpansive retract of $E$.

\section{Positively Homogeneous NoneXpansive Mappings}

Let $E$ be a Banach space and let $C$ be a closed convex cone of $E$. Then, a mapping $T: C \rightarrow C$ is called positively homogeneous if $T(\alpha x)=\alpha T(x)$ for all $x \in C$ and $\alpha>0$. In this section, we prove that a nonexpansive mapping $T: C \rightarrow C$ under an appropriate condition is generalized nonexpansive. Before proving it, we prove the following lemma.

Lemma 3.1. Let $E$ be a Banach space and let $C$ be a closed convex cone of $E$. Let $T: C \rightarrow C$ be a positively homogeneous nonexpansive mapping. Then, for any $x \in C$ and $m \in F(T)$, there exists $j \in J m$ such that

$$
\langle x-T x, j\rangle \leq 0,
$$

where $J$ is the duality mapping of $E$ into $E^{*}$.

Proof. Since $0 \in C$ and $\frac{1}{2} T 0=T\left(\frac{1}{2} 0\right)=T 0$, we have $T 0=0$. So, $F(T) \neq \emptyset$. First, let $x \in C \backslash F(T)$ and $m \in F(T)$. Suppose $m \neq 0$. We have that for $k>0$,

$$
T(k m)=k T(m)=k m .
$$

So, we have that $\frac{1}{k} x-m \neq 0$ for all $k>0$. We have from the Hahn-Banach theorem that there exists $y_{k}^{*} \in E^{*}$ such that $\left\langle\frac{1}{k} x-m, y_{k}^{*}\right\rangle=\left\|\frac{1}{k} x-m\right\|$ and $\left\|y_{k}^{*}\right\|=1$. Then, we have that

$$
\begin{aligned}
\left\langle\frac{1}{k} T x-m, y_{k}^{*}\right\rangle & \leq\left\|\frac{1}{k} T x-m\right\|=\frac{1}{k}\|T x-k m\| \\
& \leq \frac{1}{k}\|x-k m\|=\left\|\frac{1}{k} x-m\right\| \\
& =\left\langle\frac{1}{k} x-m, y_{k}^{*}\right\rangle .
\end{aligned}
$$


So, we have $\frac{1}{k}\left\langle x-T x, y_{k}^{*}\right\rangle \geq 0$ and hence

$$
\left\langle x-T x, y_{k}^{*}\right\rangle \geq 0 .
$$

Take a net $\{k>0\}$ with $k \rightarrow \infty$ and put $x_{k}=\frac{1}{k} x-m$. Then, we have $x_{k} \rightarrow-m$. Further, since $\left\{y_{k}^{*}\right\}$ is bounded, there exists a subnet $\left\{y_{k_{\alpha}}^{*}\right\}$ of $\left\{y_{k}^{*}\right\}$ converging to some $y^{*} \in E^{*}$ in the weak* topology. Let us show that $y^{*} \in E^{*}$ satisfies $\left\langle m,-y^{*}\right\rangle=\|m\|$ and $\left\|y^{*}\right\|=1$. Since the norm of $E^{*}$ is lower semicontinuous in the weak* topology, we have

$$
\left\|y^{*}\right\| \leq \liminf _{\alpha \rightarrow \infty}\left\|y_{k_{\alpha}}^{*}\right\|=1 .
$$

On the other hand, we have that

$$
\begin{aligned}
\left|\left\langle-m, y^{*}\right\rangle-\left\|x_{k_{\alpha}}\right\|\right| & =\left|\left\langle-m, y^{*}\right\rangle-\left\langle x_{k_{\alpha}}, y_{k_{\alpha}}^{*}\right\rangle\right| \\
& \leq\left|\left\langle-m, y^{*}-y_{k_{\alpha}}^{*}\right\rangle\right|+\left|\left\langle-m-x_{k_{\alpha}}, y_{k_{\alpha}}^{*}\right\rangle\right| .
\end{aligned}
$$

Since $\left\langle-m, y^{*}-y_{k_{\alpha}}^{*}\right\rangle \rightarrow 0$ and $\left\langle-m-x_{k_{\alpha}}, y_{k_{\alpha}}^{*}\right\rangle \rightarrow 0$, we have

$$
\left\|x_{k_{\alpha}}\right\| \rightarrow-\left\langle m, y^{*}\right\rangle=\left\langle m,-y^{*}\right\rangle .
$$

Since $\left\|x_{k_{\alpha}}\right\| \rightarrow\|m\|$, we have $\left\langle m,-y^{*}\right\rangle=\|m\|$. So, we have

$$
\|m\|=\left\langle m,-y^{*}\right\rangle \leq\|m\|\left\|y^{*}\right\| .
$$

From $m \neq 0$, we have $\left\|y^{*}\right\| \geq 1$. Therefore, we have $\left\|y^{*}\right\|=1$ and $\left\langle m,-y^{*}\right\rangle=$ $\|m\|$. We also have from (3.1) that

$$
\left\langle x-T x, y^{*}\right\rangle \geq 0 .
$$

Putting $z^{*}=-y^{*}$, we have $\left\|z^{*}\right\|=1,\left\langle m, z^{*}\right\rangle=\|m\|$ and

$$
\left\langle x-T x, z^{*}\right\rangle \leq 0 .
$$

So, we have

$$
\|\| m\left\|z^{*}\right\|^{2}=\|m\|^{2}=\|m\|\left\langle m, z^{*}\right\rangle=\left\langle m,\|m\| z^{*}\right\rangle .
$$

This implies $\|m\| z^{*} \in J m$, where $J$ is the duality mapping of $E$. From (3.2), we have $\|m\|\left\langle x-T x, z^{*}\right\rangle \leq 0$ and hence

$$
\langle x-T x, j\rangle \leq 0,
$$

where $j=\|m\| z^{*} \in J m$. In the case of $m=0$, we have $\{0\}=J m$. So, we have

$$
\langle x-T x, 0\rangle=0 .
$$


From (3.3) and (3.4), we have that for any $x \in C \backslash F(T)$ and $m \in F(T)$, there exists $j \in J m$ such that

$$
\langle x-T x, j\rangle \leq 0 .
$$

In the case of $x \in F(T)$, we also have

$$
\langle x-T x, j\rangle=\langle 0, j\rangle=0,
$$

where $j \in J m$. Therefore, we have that for any $x \in C$ and $m \in F(T)$, there exists $j \in J m$ such that

$$
\langle x-T x, j\rangle \leq 0 .
$$

Using Lemma 3.1, we obtain the following theorem.

Theorem 3.2. Let $E$ be a smooth Banach space and let $C$ be a closed convex cone of $E$. Let $T: C \rightarrow C$ be a positively homogeneous nonexpansive mapping. Then, $T$ is a generalized nonexpansive mapping.

Proof. Since $0 \in F(T)$, we have that for any $x \in C$,

$$
\|T x\|=\|T x-0\| \leq\|x-0\|=\|x\| .
$$

So, we have from Lemma 3.1 that for any $x \in C$ and $m \in F(T)$,

$$
\begin{aligned}
\phi(T x, m) & =\|T x\|^{2}-2\langle T x, J m\rangle+\|m\|^{2} \\
& \leq\|x\|^{2}-2\langle x, J m\rangle+\|m\|^{2}=\phi(x, m) .
\end{aligned}
$$

Therefore, $T$ is a generalized nonexpansive mapping of $C$ into itself.

\section{Weak Convergence Theorems}

In this section, we prove a weak convergence theorem of Mann's iteration for positively homogeneous nonexpansive mappings in a Banach space. Before proving it, we obtain the following lemma.

Lemma 4.1. Let $E$ be a smooth and uniformly convex Banach space and let $C$ be a nonempty closed subset of $E$ such that $J C$ is closed and convex. Let $T: C \rightarrow C$ be a generalized nonexpansive mapping such that $F(T) \neq \emptyset$. Let $\left\{\alpha_{n}\right\}$ be a sequence of real numbers such that $0 \leq \alpha_{n}<1$ and let $\left\{x_{n}\right\}$ be a sequence in $C$ generated by $x_{1}=x \in C$ and

$$
x_{n+1}=R_{C}\left(\alpha_{n} x_{n}+\left(1-\alpha_{n}\right) T x_{n}\right), \quad \forall n \in \mathbb{N},
$$

where $R_{C}$ is a sunny generalized nonexpansive retraction of $E$ onto $C$. If $R_{F(T)}$ is a sunny generalized nonexpansive retraction of $C$ onto $F(T)$, then $\left\{R_{F(T)} x_{n}\right\}$ converges strongly to an element $z$ of $F(T)$. 
Proof. Let $m \in F(T)$. Since $R_{C}$ and $T$ are generalized nonexpansive,

$$
\begin{aligned}
\phi\left(x_{n+1}, m\right) & =\phi\left(R_{C}\left(\alpha_{n} x_{n}+\left(1-\alpha_{n}\right) T x_{n}\right), m\right) \\
& \leq \phi\left(\alpha_{n} x_{n}+\left(1-\alpha_{n}\right) T x_{n}, m\right) \\
& \leq \alpha_{n} \phi\left(x_{n}, m\right)+\left(1-\alpha_{n}\right) \phi\left(T x_{n}, m\right) \\
& \leq \alpha_{n} \phi\left(x_{n}, m\right)+\left(1-\alpha_{n}\right) \phi\left(x_{n}, m\right) \\
& =\phi\left(x_{n}, m\right) .
\end{aligned}
$$

So, $\lim _{n \rightarrow \infty} \phi\left(x_{n}, m\right)$ exists. Since $\left\{\phi\left(x_{n}, m\right)\right\}$ is bounded, $\left\{x_{n}\right\}$ and $\left\{T x_{n}\right\}$ are bounded. Define $y_{n}=R_{F(T)} x_{n}$ for all $n \in \mathbb{N}$. Since $\phi\left(x_{n+1}, m\right) \leq \phi\left(x_{n}, m\right)$ for all $m \in F(T)$, from $y_{n} \in F(T)$ we have

$$
\phi\left(x_{n+1}, y_{n}\right) \leq \phi\left(x_{n}, y_{n}\right) .
$$

From Lemma 2.9 and (4.1), we have

$$
\begin{aligned}
\phi\left(x_{n+1}, y_{n+1}\right) & =\phi\left(x_{n+1}, R_{F(T)} x_{n+1}\right) \\
& \leq \phi\left(x_{n+1}, y_{n}\right)-\phi\left(R_{F(T)} x_{n+1}, y_{n}\right) \\
& =\phi\left(x_{n+1}, y_{n}\right)-\phi\left(y_{n+1}, y_{n}\right) \\
& \leq \phi\left(x_{n+1}, y_{n}\right) \\
& \leq \phi\left(x_{n}, y_{n}\right) .
\end{aligned}
$$

So, $\phi\left(x_{n}, y_{n}\right)$ is a convergent sequence. We also have from (4.1) that for all $m \in \mathbb{N}$,

$$
\phi\left(x_{n+m}, y_{n}\right) \leq \phi\left(x_{n}, y_{n}\right) .
$$

From $y_{n+m}=R_{F(T)} x_{n+m}$ and Lemma 2.9, we have

$$
\phi\left(y_{n+m}, y_{n}\right)+\phi\left(x_{n+m}, y_{n+m}\right) \leq \phi\left(x_{n+m}, y_{n}\right) \leq \phi\left(x_{n}, y_{n}\right)
$$

and hence

$$
\phi\left(y_{n+m}, y_{n}\right) \leq \phi\left(x_{n}, y_{n}\right)-\phi\left(x_{n+m}, y_{n+m}\right) .
$$

Using Lemma 2.6, we have that

$$
g\left(\left\|y_{n+m}-y_{n}\right\|\right) \leq \phi\left(y_{n+m}, y_{n}\right) \leq \phi\left(x_{n}, y_{n}\right)-\phi\left(x_{n+m}, y_{n+m}\right),
$$

where $g:[0, \infty) \rightarrow[0, \infty)$ is a continuous, strictly increasing and convex function such that $g(0)=0$. Then, the properties of $g$ yield that $R_{F(T)} x_{n}$ converges strongly to an element $z$ of $F(T)$.

Using Lemma 4.1, we prove the following theorem. 
Theorem 4.2. Let $E$ be a smooth and uniformly convex Banach space and let $C$ be a closed convex cone of $E$ such that $J C$ is closed and convex. Let $T: C \rightarrow C$ be a positively homogeneous nonexpansive mapping. Let $\left\{\alpha_{n}\right\}$ be a sequence of real numbers such that $0 \leq \alpha_{n}<1$ and $\sum_{n=1}^{\infty} \alpha_{n}\left(1-\alpha_{n}\right)=\infty$. Then, a sequence $\left\{x_{n}\right\}$ generated by $x_{1}=x \in C$ and

$$
x_{n+1}=\alpha_{n} x_{n}+\left(1-\alpha_{n}\right) T x_{n}, \quad \forall n \in \mathbb{N}
$$

converges weakly to $z \in F(T)$. Further, if $E$ has a Fréchet differentiable norm, then $z=\lim _{n \rightarrow \infty} R x_{n}$, where $R$ is a sunny generalized nonexpansive retraction of $C$ onto $F(T)$.

Proof. Let $m \in F(T)$. Then, we have

$$
\begin{aligned}
\left\|x_{n+1}-m\right\| & =\left\|\alpha_{n} x_{n}+\left(1-\alpha_{n}\right) T x_{n}-m\right\| \\
& \leq \alpha_{n}\left\|x_{n}-m\right\|+\left(1-\alpha_{n}\right)\left\|T x_{n}-m\right\| \\
& \leq \alpha_{n}\left\|x_{n}-m\right\|+\left(1-\alpha_{n}\right)\left\|x_{n}-m\right\| \\
& =\left\|x_{n}-m\right\| .
\end{aligned}
$$

So, $\lim _{n \rightarrow \infty}\left\|x_{n}-m\right\|$ exists. Putting $\lim _{n \rightarrow \infty}\left\|x_{n}-m\right\|=c$, without loss of generality, we can assume $c \neq 0$. Using Lemma 2.1, we have that

$$
\begin{aligned}
\left\|x_{n+1}-m\right\| & =\left\|\alpha_{n} x_{n}+\left(1-\alpha_{n}\right) T x_{n}-m\right\| \\
& \leq\left\|\alpha_{n}\left(x_{n}-m\right)+\left(1-\alpha_{n}\right)\left(T x_{n}-m\right)\right\| \\
& \leq\left\|x_{n}-m\right\|\left\{1-2 \alpha_{n}\left(1-\alpha_{n}\right) \delta\left(\frac{\left\|T x_{n}-x_{n}\right\|}{\left\|x_{n}-m\right\|}\right)\right\} .
\end{aligned}
$$

Then, we obtain

$$
2 c \sum_{n=1}^{\infty} \alpha_{n}\left(1-\alpha_{n}\right) \delta\left(\frac{\left\|T x_{n}-x_{n}\right\|}{\left\|x_{n}-m\right\|}\right) \leq\left\|x_{1}-m\right\|-c<\infty .
$$

From the assumptions of $\left\{\alpha_{n}\right\}$, we have

$$
\liminf _{n \rightarrow \infty} \delta\left(\frac{\left\|T x_{n}-x_{n}\right\|}{\left\|x_{n}-m\right\|}\right)=0
$$

Then, we have

$$
\liminf _{n \rightarrow \infty}\left\|T x_{n}-x_{n}\right\|=0 .
$$

On the other hand, we have 


$$
\begin{aligned}
& \left\|T x_{n+1}-x_{n+1}\right\| \\
= & \alpha_{n}\left\|T x_{n+1}-x_{n}\right\|+\left(1-\alpha_{n}\right)\left\|T x_{n+1}-T x_{n}\right\| \\
\leq & \alpha_{n}\left(\left\|T x_{n+1}-x_{n+1}\right\|+\left\|x_{n+1}-x_{n}\right\|\right)+\left(1-\alpha_{n}\right)\left\|T x_{n+1}-T x_{n}\right\| \\
\leq & \alpha_{n}\left\|T x_{n+1}-x_{n+1}\right\|+\left\|x_{n+1}-x_{n}\right\| \\
= & \alpha_{n}\left\|T x_{n+1}-x_{n+1}\right\|+\left(1-\alpha_{n}\right)\left\|T x_{n}-x_{n}\right\| .
\end{aligned}
$$

Then, we have $\left\|T x_{n+1}-x_{n+1}\right\| \leq\left\|T x_{n}-x_{n}\right\|$. So, we obtain that

$$
\lim _{n \rightarrow \infty}\left\|T x_{n}-x_{n}\right\|=\liminf _{n \rightarrow \infty}\left\|T x_{n}-x_{n}\right\|=0 .
$$

Since $E$ is reflexive and $\left\{x_{n}\right\}$ is bounded, there exists a subsequence $\left\{x_{n_{i}}\right\}$ of $\left\{x_{n}\right\}$ such that $x_{n_{i}} \rightarrow v$ for some $v \in C$. Since $E$ is uniformly convex and $\lim _{n \rightarrow \infty}\left\|T x_{n}-x_{n}\right\|=0$, we have from Lemma 2.2 that $v$ is a fixed point of $T$. Let $\left\{x_{n_{i}}\right\}$ and $\left\{x_{n_{j}}\right\}$ be two subsequences of $\left\{x_{n}\right\}$ such that $x_{n_{i}} \rightarrow u$ and $x_{n_{j}} \rightarrow v$. We know that $u, v \in F(T)$. We know from Theorem 3.2 that $T$ is a generalized nonexpansive mapping of $C$ into itself. Then, we have from the convexity of $\|\cdot\|^{2}$ that for any $m \in F(T)$,

$$
\begin{aligned}
\phi\left(x_{n+1}, m\right) & =\phi\left(\alpha_{n} x_{n}+\left(1-\alpha_{n}\right) T x_{n}, m\right) \\
& \leq \alpha_{n} \phi\left(x_{n}, m\right)+\left(1-\alpha_{n}\right) \phi\left(T x_{n}, m\right) \\
& \leq \alpha_{n} \phi\left(x_{n}, m\right)+\left(1-\alpha_{n}\right) \phi\left(x_{n}, m\right) \\
& =\phi\left(x_{n}, m\right)
\end{aligned}
$$

for all $n \in \mathbb{N}$. Then, $\lim _{n \rightarrow \infty} \phi\left(x_{n}, m\right)$ exists. Put

$$
a=\lim _{n \rightarrow \infty}\left(\phi\left(x_{n}, u\right)-\phi\left(x_{n}, v\right)\right) .
$$

Since $\phi\left(x_{n}, u\right)-\phi\left(x_{n}, v\right)=2\left\langle x_{n}, J v-J u\right\rangle+\|u\|^{2}-\|v\|^{2}$, we have

$$
a=2\langle u, J v-J u\rangle+\|u\|^{2}-\|v\|^{2}
$$

and

$$
a=2\langle v, J v-J u\rangle+\|u\|^{2}-\|v\|^{2} .
$$

From these equalities, we obtain

$$
\langle u-v, J u-J v\rangle=0 .
$$


Since $J$ is strictly monotone, it follows that $u=v$; see [44]. Therefore, $\left\{x_{n}\right\}$ converges weakly to an element $u$ of $F(T)$. On the other hand, we know from Lemma 4.1 that $\left\{R_{F(T)} x_{n}\right\}$ converges strongly to an element $z$ of $F(T)$. From Lemma 2.9, we also have

$$
\left\langle x_{n}-R_{F(T)} x_{n}, J R_{F(T)} x_{n}-J u\right\rangle \geq 0 .
$$

Since $E$ has a Fréchet differentiable norm, the duality mapping $J$ is norm-to-norm continuous. So, we have $\langle u-z, J z-J u\rangle \geq 0$. Since $J$ is monotone, we also have $\langle u-z, J z-J u\rangle \leq 0$. So, we have $\langle u-z, J z-J u\rangle=0$. Since $E$ is strictly convex, we have $z=u$. This completes the proof.

\section{Strong Convergence Theorems}

In this section, we prove a strong convergence theorem by a hybrid method called the shrinking projection method for positively homogeneous nonexpansive mappings in a Banach space.

Theorem 5.1. Let $E$ be a uniformly convex Banach space which has a Fréchet differentiable norm. Let $T: E \rightarrow E$ be a positively homogeneous nonexpansive mapping. Let $\left\{\alpha_{n}\right\}$ be a sequence of real numbers such that $0 \leq \alpha_{n} \leq a<1$. Let $\left\{x_{n}\right\}$ be a sequence generated by $x_{1}=x \in E, C_{1}=E$ and

$$
\left\{\begin{array}{l}
u_{n}=\alpha_{n} x_{n}+\left(1-\alpha_{n}\right) T x_{n} \\
C_{n+1}=\left\{z \in C_{n}: \phi\left(u_{n}, z\right) \leq \phi\left(x_{n}, z\right)\right\} \\
x_{n+1}=R_{C_{n+1}} x, \quad \forall n \in \mathbb{N}
\end{array}\right.
$$

where $R_{C_{n+1}}$ is the sunny generalized nonexpansive retraction of $E$ onto $C_{n+1}$. Then, $\left\{x_{n}\right\}$ converges strongly to $z=R_{F(T)} x$, where $R_{F(T)}$ is the sunny generalized nonexpansive retraction of $E$ onto $F(T)$.

Proof. We know that $T$ is a generalized nonexpansive mapping of $E$ into itself. So, we have from Lemma 2.13 that $F(T)$ is a sunny generalized nonexpansive retract of $E$. We shall show that $J C_{n}$ are closed and convex, and $F(T) \subset C_{n}$ for all $n \in \mathbb{N}$. It is obvious from the assumption that $J C_{1}=J E=E^{*}$ is closed and convex, and $F(T) \subset C_{1}$. Suppose that $J C_{k}$ is closed and convex, and $F(T) \subset C_{k}$ for some $k \in \mathbb{N}$. From the definition of $\phi$, we know that for $z \in C_{k}$,

$$
\begin{aligned}
& \phi\left(u_{k}, z\right) \leq \phi\left(x_{k}, z\right) \\
\Longleftrightarrow & \left\|u_{k}\right\|^{2}-\left\|x_{k}\right\|^{2}-2\left\langle u_{k}-x_{k}, J z\right\rangle \leq 0 .
\end{aligned}
$$


So, $J C_{k+1}$ is closed and convex. If $z \in F(T) \subset C_{k}$, then we have

$$
\begin{aligned}
\phi\left(u_{n}, z\right) & =\phi\left(\alpha_{n} x_{n}+\left(1-\alpha_{n}\right) T x_{n}, z\right) \\
& \leq \alpha_{n} \phi\left(x_{n}, z\right)+\left(1-\alpha_{n}\right) \phi\left(T x_{n}, z\right) \\
& \leq \alpha_{n} \phi\left(x_{n}, z\right)+\left(1-\alpha_{n}\right) \phi\left(x_{n}, z\right) \\
& =\phi\left(x_{n}, z\right) .
\end{aligned}
$$

Hence, we have $z \in C_{k+1}$. By induction, we have that $J C_{n}$ are closed and convex, and $F(T) \subset C_{n}$ for all $n \in \mathbb{N}$. Since $J C_{n}$ is closed and convex, from Lemma 2.8 there exists a unique sunny generalized nonexpansive retraction $R_{C_{n}}$ of $E$ onto $C_{n}$. We also know from Lemma 2.10 that such $R_{C_{n}}$ is denoted by $J^{-1} \Pi_{J C_{n}} J$, where $J$ is the duality mapping of $E$ and $\Pi_{J C_{n}}$ is the generalized projection of $E$ onto $J C_{n}$. Thus, $\left\{x_{n}\right\}$ is well-defined.

Since $\left\{J C_{n}\right\}$ is a nonincreasing sequence of nonempty closed convex subsets of $E^{*}$ with respect to inclusion, it follows that

$$
\emptyset \neq J F(T) \subset \text { M- } \lim _{n \rightarrow \infty} J C_{n}=\cap_{n=1}^{\infty} J C_{n} .
$$

Put $C_{0}^{*}=\cap_{n=1}^{\infty} J C_{n}$. Then, by Theorem 2.7 we have that $\left\{\Pi_{J C_{n+1}} J x\right\}$ converges strongly to $x_{0}^{*}=\Pi_{C_{0}^{*}} J x$. Since $E^{*}$ has a Fréchet differencial norm, $J^{-1}$ is continuous. So, we have

$$
x_{n+1}=R_{n+1} x=J^{-1} \Pi_{J C_{n+1}} J x \rightarrow J^{-1} x_{0}^{*} .
$$

To complete the proof, it is sufficient to show that $J^{-1} x_{0}^{*}=R_{F(T)} x$.

Since $x_{n}=R_{C_{n}} x$ and $x_{n+1}=R_{C_{n+1}} x \in C_{n+1} \subset C_{n}$, we have from Lemma 2.9 and (2.2) that

$$
\begin{aligned}
0 & \leq 2\left\langle x-x_{n}, J x_{n}-J x_{n+1}\right\rangle \\
& =\phi\left(x, x_{n+1}\right)-\phi\left(x, x_{n}\right)-\phi\left(x_{n}, x_{n+1}\right) \\
& \leq \phi\left(x, x_{n+1}\right)-\phi\left(x, x_{n}\right) .
\end{aligned}
$$

So, we get that

$$
\phi\left(x, x_{n}\right) \leq \phi\left(x, x_{n+1}\right) .
$$

Further, since $x_{n}=R_{C_{n}} x$ and $z \in F(T) \subset C_{n}$, from Lemma 2.12 we have

$$
\phi\left(x, x_{n}\right) \leq \phi(x, z)
$$


So, we have that $\lim _{n \rightarrow \infty} \phi\left(x, x_{n}\right)$ exists. This implies that $\left\{x_{n}\right\}$ is bounded. Hence, $\left\{u_{n}\right\}$ and $\left\{T x_{n}\right\}$ are also bounded. From

$$
\begin{aligned}
\phi\left(x_{n}, x_{n+1}\right) & =\phi\left(R_{C_{n}} x, x_{n+1}\right) \\
& =\phi\left(x, x_{n+1}\right)-\phi\left(x, R_{C_{n}} x\right) \\
& =\phi\left(x, x_{n+1}\right)-\phi\left(x, x_{n}\right) \rightarrow 0,
\end{aligned}
$$

we have that

$$
\phi\left(x_{n}, x_{n+1}\right) \rightarrow 0 .
$$

From $x_{n+1} \in C_{n+1}$, we have that $\phi\left(u_{n}, x_{n+1}\right) \leq \phi\left(x_{n}, x_{n+1}\right)$. So, we get that $\phi\left(u_{n}, x_{n+1}\right) \rightarrow 0$. Using Lemma 2.5 , we have

$$
\lim _{n \rightarrow \infty}\left\|u_{n}-x_{n+1}\right\|=\lim _{n \rightarrow \infty}\left\|x_{n}-x_{n+1}\right\|=0 .
$$

So, we have

$$
\left\|u_{n}-x_{n}\right\| \leq\left\|u_{n}-x_{n+1}\right\|+\left\|x_{n+1}-x_{n}\right\| \rightarrow 0 .
$$

Since $\left\|x_{n}-u_{n}\right\|=\left\|x_{n}-\alpha_{n} x_{n}-\left(1-\alpha_{n}\right) T x_{n}\right\|=\left(1-\alpha_{n}\right)\left\|x_{n}-T x_{n}\right\|$ and $0 \leq \alpha_{n} \leq a<1$, we have that

$$
\left\|T x_{n}-x_{n}\right\| \rightarrow 0 .
$$

Since $x_{n+1} \rightarrow J^{-1} x_{0}^{*}$ and $T$ is continuous, we have $J^{-1} x_{0}^{*} \in F(T)$. that

Put $z_{0}=R_{F(T)} x$. Since $z_{0}=R_{F(T)} x \subset C_{n+1}$ and $x_{n+1}=R_{C_{n+1}} x$, we have

$$
\phi\left(x, x_{n+1}\right) \leq \phi\left(x, z_{0}\right)
$$

So, we have that

$$
\begin{aligned}
\phi\left(x, J^{-1} x_{0}^{*}\right) & =\|x\|^{2}-2\left\langle x, x_{0}^{*}\right\rangle+\left\|J^{-1} x_{0}^{*}\right\|^{2} \\
& =\lim _{n \rightarrow \infty}\left(\|x\|^{2}-2\left\langle x, J x_{n}\right\rangle+\left\|x_{n}\right\|^{2}\right) \\
& =\lim _{n \rightarrow \infty} \phi\left(x, x_{n}\right) \\
& \leq \phi\left(x, z_{0}\right) .
\end{aligned}
$$

So, we get $z_{0}=J^{-1} x_{0}^{*}$. Hence, $\left\{x_{n}\right\}$ converges strongly to $z_{0}$. This completes the proof.

Using Theorem 5.1, we prove a strong convergence theorem for linear contractive mappings in a Banach space. 
Theorem 5.2. Let $E$ be a uniformly convex Banach space which has a Fréchet differentiable norm. Let $T: E \rightarrow E$ be a linear contractive mapping. Let $\left\{\alpha_{n}\right\}$ be a sequence of real numbers such that $0 \leq \alpha_{n} \leq a<1$. Let $\left\{x_{n}\right\}$ be a sequence generated by $x_{1}=x \in E, C_{1}=E$ and

$$
\left\{\begin{array}{l}
u_{n}=\alpha_{n} x_{n}+\left(1-\alpha_{n}\right) T x_{n} \\
C_{n+1}=\left\{z \in C_{n}: \phi\left(u_{n}, z\right) \leq \phi\left(x_{n}, z\right)\right\} \\
x_{n+1}=R_{C_{n+1}} x, \quad \forall n \in \mathbb{N}
\end{array}\right.
$$

where $R_{C_{n+1}}$ is the sunny generalized nonexpansive retraction of $E$ onto $C_{n+1}$. Then, $\left\{x_{n}\right\}$ converges strongly to $z=R_{F(T)} x$, where $R_{F(T)}$ is the sunny generalized nonexpansive retraction of $E$ onto $F(T)$.

Proof. A linear contractive mapping $T: E \rightarrow E$ is positively homogeneous and nonexpansive. So, using Theorem 5.1, we obtain the desired result.

\section{ACKNOWLEDGMENTS}

The research of the first author and the second author was partially supported by Grant-in-Aid for Scientific Research No. 19540167 from Japan Society for the Promotion of Science and by the grant NSC 98-2115-M-110-001, respectively.

\section{REFERENCES}

1. Y. I. Alber, Metric and generalized projections in Banach spaces: Properties and applications, in Theory and Applications of Nonlinear Operators of Accretive and Monotone Type (A. G. Kartsatos Ed.), Marcel Dekker, New York, 1996, pp. 15-20.

2. Y. I. Alber and S. Reich, An iterative method for soling a class of nonlinear operator equations in Banach spaces, PanAmer. Math. J., 4 (1994), 39-54.

3. K. Aoyama, F. Kohsaka and W. Takahashi, Three generalizations of firmly nonexpansive mappings: Their relations and continuity properties, J. Nonlinear Convex Anal., 10 (2009), 131-147.

4. K. Aoyama and W. Takahashi, Strong convergence theorems for a family of relatively nonexpansive mappings in Banach spaces, Fixed Point Theory, 8 (2007), 143-160.

5. F. E. Browder, Nonexpansive nonlinear operators in a Banach space, Proc. Nat. Acad. Sci. USA, 54 (1965), 1041-1044.

6. F. E. Browder, Semicontractive and semiaccretive nonlinear mappings in Banach spaces, Bull. Amer. Math. Soc., 74 (1968), 660-665.

7. R. E. Bruck, On the convex approximation property and the asymptotic behaviour of nonlinear contractions in Bnach spaces, Israel J. Math., 38 (1981), 304-314. 
8. J. Diestel, Geometry of Banach spaces, Selected Topics, Lecture Notes in Mathematics, 485, Springer, Berlin, 1975.

9. T. Honda, T. Ibaraki and W. Takahashi, Duality theorems and convergence theorems for nonlineaqr mappings in Banach spaces, Int. J. Math. Statis., 6 (2010), 46-64.

10. T. Honda and W. Takahashi, Nonlinear projections and generalized conditional expectations in Banach spaces, Taiwanese J. Math., to appear.

11. T. Honda and W. Takahashi, Norm one linear projections and generalized conditional expectations in Banach spaces, Sci. Math. Jpn, 69 (2009), 303-313.

12. T. Ibaraki, Y. Kimura and W. Takahashi, Convergence theorems for generalized projections and maximal monotone operators in Banach spaces, Abst. Appl. Anal., 2003 (2003), 621-629.

13. T. Ibaraki and W. Takahashi, Weak and strong convergence theorems for new resolvents of maximal monotone operators in Banach spaces, Advances in Mathematical Economics, 10 (2007), 51-64.

14. T. Ibaraki and W. Takahashi, A new projection and convergence theorems for the projections in Banach spaces, J. Approx. Theory, 149 (2007), 1-14.

15. T. Ibaraki and W. Takahashi, Generalized nonexpansive mappings and a proximaltype algorithm in Banach spaces, Contemp. Math., to appear.

16. H. Iiduka and W. Takahashi, Weak convergence theorem by Cesaro means for nonexpansive mappings and inverse-strongly monotone mappings, J. Nonlinear Convex Anal., 7 (2006), 105-113.

17. S. Itoh and W. Takahashi, The common fixed point theory of singlevalued mappings and multivalued mappings, Pacific J. Math., 79 (1978), 493-508.

18. S. Kamimura, F. Kohsaka and W. Takahashi, Weak and strong convergence theorems for maximal monotone operators in a Banach space, Set-Valued Anal., 12 (2004), 417-429.

19. S. Kamimura and W. Takahashi, Approximating solutions of maximal monotone operators in Hilbert spaces, J. Approx. Theory, 106 (2000), 226-240.

20. S. Kamimura and W. Takahashi, Weak and strong convergence of solutions to accretive operator inclusions and applications, Set-Valued Anal., 8 (2000), 361-374.

21. S. Kamimura and W. Takahashi, Strong convergence of a proximal-type algorithm in a Banach apace, SIAM J. Optim., 13 (2002), 938-945.

22. F. Kohsaka and W. Takahashi, Strong convergence of an iterative sequence for maximal monotone operators in a Banach space, Abstr. Appl. Anal., 2004 (2004), 239249.

23. F. Kohsaka and W. Takahashi, Weak and strong convergence theorems for minimax problems in Banach spaces, in Nonlinear Analysis and Convex Analysis (W. Takahashi and T. Tanaka, Eds.), Yokohama Publishers, 2004, pp. 203-216. 
24. F. Kohsaka and W. Takahashi, Generalized nonexpansive retractions and a proximaltype algorithm in Banach spaces, J. Nonlinear Convex Anal., 8 (2007), 197-209.

25. F. Kohsaka and W. Takahashi, Existence and approximation of fixed points of firmly nonexpansive-type mappings in Banach spaces, SIAM J. Optim., 19 (2008), 824-835.

26. F. Kohsaka and W. Takahashi, Fixed point theorems for a class of nonlinear mappings related to maximal monotone operators in Banach spaces, Arch. Math., 91 (2008), 166-177.

27. S. Matsushita and W. Takahashi, Weak and strong convergence theorems for relatively nonexpansive mappings in Banach spaces, Fixed Point Theory Appl., 2004 (2004), $37-47$.

28. S. Matsushita and W. Takahashi, A strong convergence theorem for relatively nonexpansive mappings in a Banach space, J. Approx. Theory, 134 (2005), 257-266.

29. S. Matsushita and W. Takahashi, Approximating fixed points of nonexpansive mappings in a Banach space by metric projections, Applied Math. Comput., 196 (2008), 422-425.

30. U. Mosco, convergence of convex sets and of solutions of variational inequalities, Adv. Math., 3 (1969), 510-585.

31. A. Moudafi, Weak convergence theorems for nonexpansive mappings and equilibrium problems, J. Nonlinear Convex Anal., 9 (2008), 37-43.

32. A. Moudafi and M. Théra, Proximal and dynamical approaches to equilibrium problems, Lecture Notes in Economics and Mathematical Systems, 477, Springer, 1999, pp. 187-201.

33. K. Nakajo and W. Takahashi, Strong convergence theorems for nonexpansive mappings and nonexpansive semigroups, J. Math. Anal. Appl., 279 (2003), 372-379.

34. S. Ohsawa and W. Takahashi, Strong convergence theorems for resolvento of maximal monotone operator, Arch. Math., 81 (2003), 439-445.

35. Z. Opial, Weak covergence of the sequence of successive approximations for nonexpansive mappings, Bull. Amer. Math. Soc., 73 (1967), 591-597.

36. S. Reich, Weak convergence theorems for nonexpansive mappings in Banach spaces, J. Math. Anal. Appl., 67 (1979), 274-276.

37. S. Reich, Strong convergence theorems for resolvents of accretive operators in Banach spaces, J. Math. Anal. Appl., 75 (1980), 287-292.

38. S. Reich, A weak convergence theorem for the alternative method with Bregman distance, in Theory and Applications of Nonlinear Operators of Accretive and Monotone Type (A. G. Kartsatos Ed.), Marcel Dekker, New York, 1996, pp. 313-318.

39. M. V. Solodov and B. F. Svaiter, Forcing strong convergence of proximal point iterations in a Hilbert space, Math. Program., 87 (2000), 189-202.

40. A. Tada and W. Takahashi, Strong convergence theorem for an equilibrium problem and a nonexpansive mapping, J. Optim. Theory Appl., 133 (2007), 359-370. 
41. S. Takahashi and W. Takahashi, Viscosity approximation methods for equilibrium problems and fixed point problems in Hilbert spaces, J. Math. Anal. Appl., 331 (2007), 506-515.

42. S. Takahashi and W. Takahashi, Strong convergence theorems for a generalized equilibrium problem anda nonexpansive mapping in a Hilbert space, Nonlinear Anal., 69 (2008), 1025-1033.

43. W. Takahashi, Iterative methods for approximation of fixed points and their applications, J. Oper. Res. Soc. Japan, 43 (2000), 87-108.

44. W. Takahashi, Nonlinear Functional Analysis, Yokohama Publishers, Yokohama, 2000.

45. W. Takahashi, Convex Analysis and Approximation of Fixed Points (Japanese), Yokohama Publishers, Yokohama, 2000.

46. W. Takahashi, Introduction to Nonlinear and Convex Analysis (Japanese), Yokohama Publishers, Yokohama, 2005.

47. W. Takahashi, Viscosity approximation methods for resolvents of accretive operators in Banach spaces, J. Fixed Point Theory Appl., 1 (2007), 135-147.

48. W. Takahashi, Proximal point algorithms and four resolvents of nonlinear operators of monotone type in Banach spaces, Taiwanese J. Math., 12 (2008), 1883-1910.

49. W. Takahashi, Viscosity approximation methods for countable families of nonexpansive mappings in Banach spaces, Nonlinear Anal., 70 (2009), 719-734.

50. W. Takahashi, Fixed point theorems for new nonexpansive mappings in a Hilbert space, J. Nonlinear Convex Anal., 11 (2010), 78-88.

51. W. Takahashi and G. E. Kim, Approximating fixed points of nonexpansive mappings in Banach spaces, Math. Japon., 48 (1998), 1-9.

52. W. Takahashi, Y. Takeuchi and R. Kubota, Strong convergence theorems by hybrid methods for families of nonexpansive mappings in Hilbert spaces, J. Math. Anal. Appl., 341 (2008), 276-286.

53. W. Takahashi and M. Toyoda, Weak convergence theorems for nonexpansive mappings and monotone mappings, J. Optim. Theory Appl., 118 (2003), 417-428.

54. W. Takahashi and Y. Ueda, On Reich's strong convergence theorems for resolvents of accretive operators, J. Math. Anal. Appl., 104 (1984), 546-553.

55. W. Takahashi and J. C. Yao, Fixed point theorems and ergodic theorems for nonlinear mappings in a Hilbert space, Taiwanese J. Math., to appear.

56. H. K. Xu, Inequalities in Banach spaces with applications, Nonlinear Anal., 16 (1991), 1127-1138. 
Wataru Takahashi

Department of Applied Mathematics

National Sun Yat-sen University

Kaohsiung 80424

Taiwan

E-mail: wataru@is.titech.ac.jp

Jen-Chih Yao

Center for General Education

Kaohsiung Medical University

Kaohsiung 80707, Taiwan

E-mail: yaojc@kmu.edu.tw 